\title{
Attentional prioritization to contextually new objects
}

\author{
HIROKAZU OGAWA and TAKATSUNE KUMADA \\ National Institute of Advanced Industrial Science and Technology, Tsukuba, Japan
}

\begin{abstract}
Using a hybrid paradigm of a contextual cuing task and a probe detection task, we tested whether or not contextually new objects can be prioritized in visual search. After several hundred visual search practice trials with repeated and nonrepeated layouts, an additional distractor that was contextually new was presented in the repeated layouts. The results showed that detection of probe dots appearing at the location of a search target was faster in the repeated than in the nonrepeated layouts. More importantly, detection of probe dots at the new object locations was as fast as that of probe dots at the target locations in repeated layouts, suggesting that the attentional system implicitly prioritizes the processing of a location where a change in contextual information has occurred.
\end{abstract}

Allocating visual attention to potentially important locations and objects in a visual scene is a critical function of the visual system. Extensive literature in which visual search tasks were used has reported the mechanisms controlling visual attention and demonstrated that the visual system can utilize an observer's memory or knowledge acquired through past experience to guide attention to ecologically relevant information in the scene (e.g., Chun \& Jiang, 1998; Klein, 1988; Maljkovic \& Nakayama, 2000; Watson \& Humphreys, 1997).

One recent demonstration showed that a memory-based process facilitates the attentional acquisition of the relevant information (Chun \& Jiang, 1998). Chun and Jiang had participants perform hundreds of inefficient visual search tasks that involved serial deployments of attention. In half of the trials, targets were embedded in configurations of distractors that were repeated throughout the entire experiment (repeated layouts), whereas in the other half of the trials targets were presented in novel configurations of distractors (nonrepeated layouts). The target always appeared in the same location in a given configuration. The results showed that the search reaction time (RT) for targets was shorter in the repeated layouts than in the nonrepeated layouts, and the difference in RT became greater as the experiment progressed. This benefit of repeated spatial layouts of displays is referred to as the contextual cuing effect. The contextual cuing effect occurs without an awareness of learning or an explicit memory of repeated layouts. When participants were asked to discriminate the repeated layouts from the nonrepeated layouts, they

This research was supported by research fellowships to H.O. from the Japan Society for the Promotion of Science for Young Scientists. Correspondence concerning this article should be addressed to H. Ogawa, Cognitive Science Research Group, Research Center for Advanced Science and Technology, University of Tokyo, 4-6-1 Komaba, Meguro-ku, Tokyo 153-8904, Japan (e-mail: ogawa@fennel.rcast.u-tokyo.ac.jp). could not perform the discrimination above chance level even though the contextual cuing effect was present in the repeated layouts. These results were interpreted to mean that the participants implicitly learned the contingency between locations of targets and those of distractors as contextual information, which guided visual attention to the potential target locations when previously learned layouts were presented.

The contextual guidance of visual attention is considered to be ecologically valid in most cases because the visual environment that we encounter in our everyday life tends to contain regularities (Chun, 2000; Fiser \& Aslin, 2001, 2002; Reber, 1989; Saffran, Aslin, \& Newport, 1996). However, contextual information is not always invariant over time. Objects may move to other locations, or objects that were not present in the past may appear in the scene. Thus, in addition to contextual guidance of attention to locations where targets were presented in the past, it is ecologically useful to allocate attention to a new object and to update contextual information if necessary, because new objects potentially tend to be relevant for survival, food seeking, and so on. Is visual attention guided toward a location of such a contextually new object when it appears in a learned display?

Although there is substantial evidence that attention is involuntarily guided or captured by the abrupt appearance of an object in a display (see, e.g., Hillstrom \& Yantis, 1994; Jonides \& Yantis, 1988; Theeuwes, 1994; Yantis, 1993; Yantis \& Jonides, 1984, 1990), less is known regarding whether or not contextually new objects guide attention to their locations. To the best of our knowledge, this issue has been examined in only a few studies (Peterson \& Kramer, 2001a, 2001b). Peterson and Kramer (2001b) examined whether or not contextual cuing is modulated when attention is captured by an abrupt-onset distractor in a display. In their experiment, a set of placeholders to mark future locations of search items was presented for $747 \mathrm{msec}$, followed by a search display with a repeated or 
nonrepeated layout. After hundreds of learning trials with repeated and nonrepeated layouts, an additional distractor was presented simultaneously with a search display at a location that was not previously occupied by a placeholder. Since an additional distractor abruptly appeared at a location where no search items had been present during learning, it would serve as an object that is both contextually and perceptually new in repeated layouts. The results showed that the increase in search RT caused by abrupt onsets was smaller in the repeated than in the nonrepeated layouts, suggesting that contextual cuing suppressed the automatic allocation of attention to abrupt-onset distractors.

At first glance, Peterson and Kramer's (2001b) results appear to contradict the notion that contextual information guides attention toward contextually new objects. However, we would argue that the results were due to the presentation of the placeholders that allowed contextual guidance of attention toward the target location before the appearance of a new object. Thus, before the presentation of an onset distractor, attention was guided to a target location, resulting in the prevention of attentional capture by an onset distractor. This argument is consistent with previous studies indicating that attentional capture by abrupt onsets fails to occur when attention is focused elsewhere in advance (Koshino, Warner, \& Juola, 1992; Theeuwes, Kramer, Halm, \& Irwin, 1998; Yantis \& Jonides, 1990). We would also argue that because an abrupt onset of objects is in itself a very strong perceptual cue to guide attention toward their locations, it might interact with attentional guidance by contextual cuing. Under such circumstances, it may be difficult to examine the effect of attentional guidance to contextually new objects under the presentation of abrupt-onset objects.

In the present study, we examined whether or not contextually new objects that do not involve a perceptual cue by abrupt onsets guide visual attention. Participants engaged in a learning session that consisted of 540 familiarization trials with repeated and nonrepeated layouts, followed by a test session with the learned layouts plus a distractor item that had never appeared in displays of the learning session and was incongruent with the contextual information learned in repeated layouts. To measure attentional allocation to the contextually new objects directly, we adopted a hybrid paradigm of a visual search task and a probe detection task that allows the direct measurement of attentional allocation (see, e.g., Kim \& Cave, 1995; Klein, 1988; Watson \& Humphreys, 2000). In all trials of the learning session and in half of the trials of the test session, the participants performed a visual search task. In the other half of the trials in the test session, a probe task was presented in which the participants had to detect the presence of a small dot. The probe dot appeared at the location of a learned target, a learned distractor, or a new distractor that was added in the test session. If contextually new objects guide attention to their locations, RTs to probe dots on additional distractors in the repeated layout should be faster than those to probe dots on other distractors.

\section{METHOD}

\section{Participants}

Thirty-one paid volunteers (17 males and 14 females, 19-32 years of age) participated after giving informed consent. All had normal or corrected-to-normal visual acuity and normal color vision. None of the participants was aware of the purpose of this study.

\section{Apparatus and Stimuli}

The stimuli were presented on a Sony Trinitron monitor $(80-\mathrm{Hz}$ refresh rate). The experiment was programmed using MATLAB with Psychophysics Toolbox extensions (Brainard, 1997; Pelli, 1997). A standard keyboard was used as a response device. The viewing distance was approximately $60 \mathrm{~cm}$. The display elements were eight (learning session) or nine (test session) white outlined squares $\left(1.0^{\circ} \times 1.0^{\circ}\right)$, each of which had a gap $\left(0.12^{\circ}\right)$ on one side (see Figure 1). The target had a gap on either the left or the right side of the square, and each distractor had a gap on the top or bottom. The displays consisted of one target and seven (learning session) or eight (test session) distractors. All search items were randomly placed in the cells of a $5 \times 7$ virtual matrix subtending $12.5^{\circ} \times 12.5^{\circ}$ of visual angle with some random jitters within the cells. The random jitters were constant across the repetitions.

At the beginning of the experiment, 18 target locations were selected from the matrix and paired with configurations of seven distractors. These 18 layouts were presented repeatedly throughout the experimental sessions in both search and probe trials (repeated layouts). In half of the trials of a session, the repeated layouts were presented. In the other half of the trials, displays were presented in which the same 18 target locations used in the repeated layouts were paired with a configuration of seven distractors newly generated in each trial (nonrepeated layouts). The target locations of repeated layouts were identical to those of nonrepeated layouts, so that any learning observed for repeated layouts could not be attributed to the learning of target locations per se.

\section{Design and Procedure}

The participants first engaged in a learning session and then in a test session. In the learning session, the participants performed only a search task. In the search trials, the participants were asked to search for a target among the distractors and to indicate the location of the gap in the target as quickly and accurately as possible. They were instructed to press, with the right index and middle fingers, respectively, the " $<$ " key if the target had a gap on the left side and the " $>$ " key if the target had a gap on the right side. The target was present in all trials.

The learning session consisted of 540 trials. There were 270 trials for repeated layouts, resulting in 15 presentations of each repeated layout. In the remaining 270 trials, nonrepeated layouts were presented. In the learning session, data were collapsed into five epochs, with each epoch consisting of data from 108 search trials (18 repeated layouts $\times 3$ presentations, plus 54 nonrepeated layouts).

In all trials of the test session, a distractor that had never been presented in the learning session was added to the display. Thus, in the test session the displays consisted of one target and eight distractors. The additional distractor was identical to the other distractors in appearance, but its location changed in each presentation in both the repeated and nonrepeated layouts. Since all distractors in the nonrepeated layouts were presented in new locations in each trial, the additional distractor served as a contextually new object only in the repeated layouts.

In half of the trials of the test session, a visual search task, which was the same as that used in the learning session, was presented. In the remaining trials, the probe detection task was presented. In the probe detection trials, the array of search items was accompanied by a $1000-\mathrm{Hz}$ tone that lasted $50 \mathrm{msec}$. The tone indicated that the 


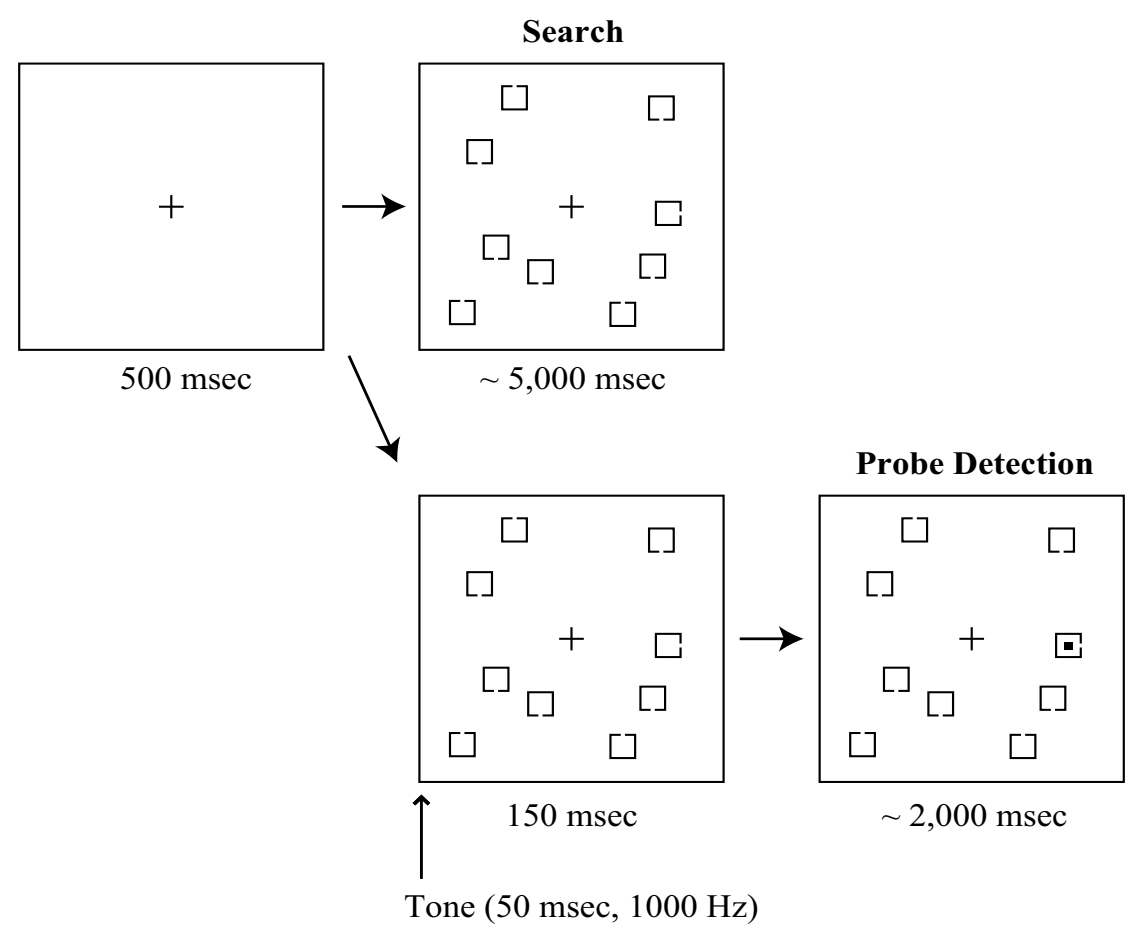

\begin{abstract}
Figure 1. Sequence of frames on a given trial. The display depicts the sequence of frames of a test session trial, which consisted of a target and seven distractors, one of which had never appeared in a learning session. The detection frame depicts a trial with a probe dot at the location of the search target (target probe). The actual stimuli were white against a black background.
\end{abstract}

participant should detect a small probe dot that was presented on one of the search items instead of searching for the target. The stimulus onset asynchrony between the onset of search items and that of the probe dot was $150 \mathrm{msec}$. The participants were instructed to press the " $\mathrm{C}$ " key with the left index finger as soon as they could detect the probe dot in a trial with a tone presentation (go/no-go task). A prolonged low-pitch tone was given after the participants' response as feedback for errors in both search trials and probe trials. The order of trials was randomized so that the participants could not predict whether the next trial would be a search trial or a probe trial.

The test session consisted of 396 search trials and 396 probe detection trials. Of the 396 trials for the search task, there were 198 trials with repeated layouts and 198 trials with nonrepeated layouts. The 18 repeated layouts were identical to the layouts used in the learning session except for the presentation of an additional distractor, and each layout was presented repeatedly (11 times) throughout the search tasks in the test session. In the probe detection task as well, there were 198 trials with repeated layouts and 198 trials with nonrepeated layouts. The probe dot was presented at the location of a target, one of the previously presented distractors, or the additional distractor. The 198 probe trials for the repeated layout condition consisted of 18 target-probe trials, 126 old-distractor-probe trials, 18 additional-distractor-probe trials, and 36 catch trials in which no probe dot appeared.

\section{RESULTS}

The mean correct RT within each epoch was calculated for each layout condition. RTs that were shorter than $100 \mathrm{msec}$ or that exceeded the criterion set by Van Selst and Jolicœur (1994) were excluded from analysis $(0.32 \%$ of all observations). Since the overall error rate was very low for the search task $(0.21 \%)$ and the probe task $(0.95 \%)$, no further analysis of errors was performed.

\section{Search Task}

Figure 2 illustrates the mean search RTs for each layout condition as a function of epoch. A two-way ANOVA was conducted with two within-subjects factors (layout, repeated vs. nonrepeated; epoch, $1-5)$. The analysis revealed significant main effects of layout $[F(1,30)=18.69, p<$ $.001]$ and epoch $[F(4,120)=16.27, p<.001]$. Overall, RTs to targets were shorter in the repeated layouts than in the nonrepeated layouts and became shorter as the experiment progressed. There was also a significant layout $\times$ epoch interaction $[F(4,120)=4.38, p<.005]$, indicating that the difference in RT between the repeated and nonrepeated layouts was greater in later epochs than in earlier epochs of the experiment.

Figure 2 also shows RTs of the search task presented in the test session. As can be seen in the figure, there was an increase in search RTs in the test session with both layout conditions, due partly to the cost of task switching or task mixing (between the search and probe tasks) and the increase in the number of search items. Despite the fact that these costs should affect search RTs in both repeated and nonrepeated layouts, the increase in the search RT was particularly marked for repeated layouts. The observations were statistically confirmed by a two-way ANOVA with 


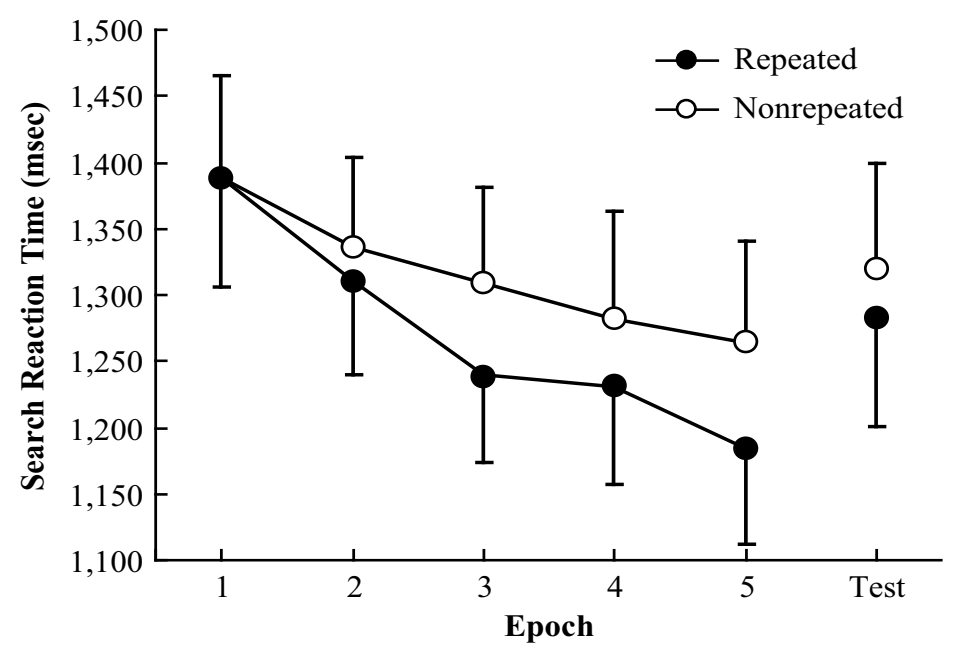

Figure 2. Mean correct search reaction times (in milliseconds) to the repeated and the nonrepeated layouts, plotted as a function of epoch. The error bars represent $95 \%$ confidence intervals.

layout (repeat vs. nonrepeated) and session (Epoch 5 vs. test session) as main factors. There were significant main effects of layout $[F(1,30)=25.89, p<.0001]$ and session $[F(1,30)=16.42, p<.001]$, and there was a significant interaction between layout and session $[F(1,30)=5.49$, $p<.05]$. Also, a post hoc comparison (Tukey's HSD test) revealed that search RTs in repeated layouts were shorter than those in nonrepeated layouts in the test session $(p<$ $.01)$.

\section{Probe Task}

Because additional distractors served as new objects in repeated layouts only, there were no probe RTs for the additional distractors in nonrepeated layouts. Figure 3 shows the mean correct RTs as a function of probe location and layout condition in the probe detection task.

To analyze separately the effect of repetition of layouts on RTs for probes presented at the location of the learned target and RTs for the contextually new objects, we conducted two ANOVAs. First, RTs in target- and distractorprobe trials were entered into a two-way ANOVA with layout (repeated vs. nonrepeated) and probe (target vs. distractor) as main factors. There was a significant interaction between layout and probe $[F(1,30)=6.30, p<.05]$, reflecting the fact that RTs to probes at target locations were shorter than RTs to probes at distractor locations in repeated layouts and that there was no difference in nonrepeated layouts. No main effects reached significance. Second, RTs in old-distractor and additional-distractor trials were entered into a one-factor (probe type: distractor in repeated layouts vs. distractor in nonrepeated layouts vs. additional distractor in repeated layouts) repeated measures ANOVA. The analysis showed a significant main effect $[F(2,60)=4.84, p<.005]$. Post hoc comparisons revealed that probe RTs at the location of a new object were shorter than those at the locations of other distractors with both repeated and nonrepeated layouts $(p<.05)$.
The probe RTs for distractors did not differ significantly between repeated and nonrepeated layouts.

\section{DISCUSSION}

The results showed that RTs to a probe that appeared in the target location were shorter in the repeated layouts than in the nonrepeated layouts, suggesting that contextual cuing guided attentional allocation to the learned target locations in the repeated layouts. This confirmed the contextual cuing effect of target locations using a probe detection task. More importantly, the present results clearly demonstrate that attention is guided to a contextually

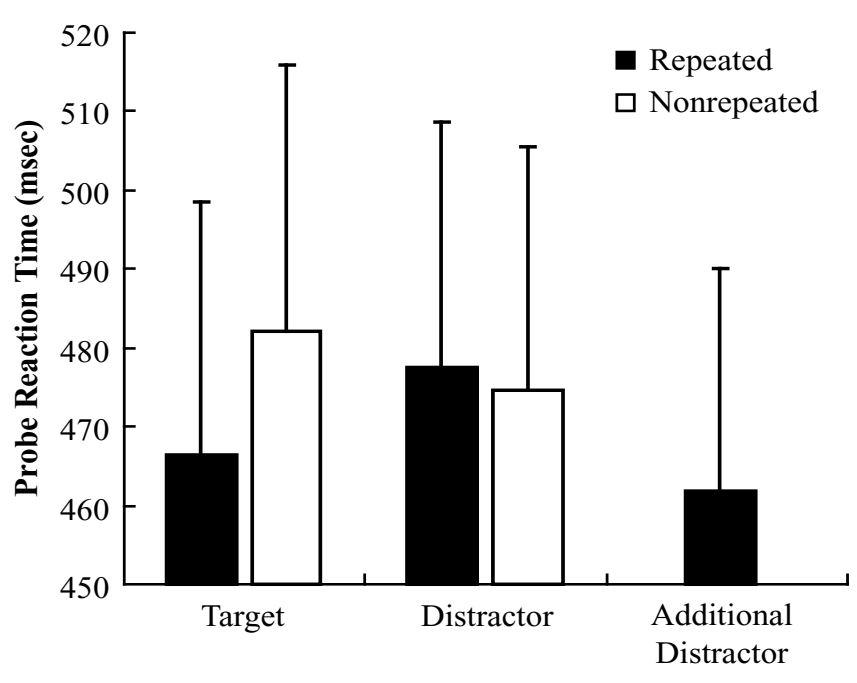

Probe Location

Figure 3. Mean correct reaction times (in milliseconds) to probes as a function of probe dot location and layout condition. The error bars represent $95 \%$ confidence intervals. 
new object. RTs to probes in new-object locations were shorter than those to other distractors in the repeated layouts. Since there was no perceptual difference between additional distractors and other distractors, this result can be attributed to contextual information's guiding attention to new objects. Furthermore, the contextual effect on the learned target location, in comparison with that on the new object location, suggests that the new object location was prioritized at the same level as the target location. These results are consistent with recent work demonstrating that attention can be allocated to multiple noncontiguous locations in parallel (see, e.g., Awh \& Pashler, 2000; Castiello \& Umiltà, 1992; Godijn \& Theeuwes, 2003; Hahn \& Kramer, 1998; Kramer \& Hahn, 1995).

The present results advance the understanding of the role of contextual information in attentional guidance. Although previous studies have repeatedly shown that the learned context of display layouts guides attention to learned target locations, the present study demonstrates a new role of context information: that of guiding attention to contextually new objects. As we discussed in the introduction, although such a role is ecologically valid, no direct evidence has been presented. Hence, the present results may shed light on the relevance of consideration from the ecological point of view on the contextual cuing effect.

An important question that arises from our results is whether attentional prioritization of new objects is an implicit process just as prioritization of a learned target in contextual cuing is. Although in the present experiment we did not test the participants' explicit awareness of repeated context, we conducted an additional experiment to examine whether or not the participants were aware of repeated layouts and an additional distractor in the layouts. In this experiment, after completing a learning session, 12 participants performed a recognition session instead of a test session. In the recognition session, layouts contained an additional distractor that did not appear in the learning session, and search items were replaced with squares that did not have gaps. The participants were asked to guess which hemifield of the display was most likely to contain the target and the additional distractor given the configuration. The results showed that performance for both the target $(55.2 \%$ and $56.8 \%$ in repeated and nonrepeated layouts, respectively) and the additional distractor (51.4\% and $52.3 \%$, respectively) were not above chance level and did not significantly differ between repeated and nonrepeated layouts, confirming that the participants did not explicitly remember repeated layouts and did not direct attention to locations of an additional distractor. Therefore, our results demonstrate that the attentional system can detect a change in learned contextual information independently of the observer's awareness, and that it allocates attention to the location to update the learned contextual information.

Attentional guidance to contextually new objects may be mediated by a mechanism unlike that for attentional guidance to learned targets. Contextual cuing to learned target locations is assumed to be based on the memory for the relationship between a repeated target location and distractor locations (or a configuration of a display) learned in learning sessions. However, in the case of guidance to new objects, there is no memory for the new object locations because the new objects did not appear in the learning sessions. Thus, attentional guidance to contextually new objects occurs without a top-down signal concerning where to direct attention and with a bottomup signal of a search display. Some researchers in attentional capture have argued that the appearance of a new object, rather than a transient signal that is induced by the onset of objects, plays a special role in attentional capture (Hillstrom \& Yantis, 1994; Yantis \& Hillstrom, 1994). The present results can be interpreted as new evidence that new objects can "capture" attention on the basis of implicit information about the appearance of an object even when there is no perceptual impression of the emergence of new objects. Obviously, future research will be needed to examine this interpretation.

Numerous studies have demonstrated top-down influences of familiarity on visual perception, including the word superiority effect (Reicher, 1969), the object superiority effect (Weisstein \& Harris, 1974), and novel pop-out (Johnston, Schwarting, \& Hawley, 1996). Among them, there is especially an apparent analogy between the new object advantage observed in the present study and the semantic inconsistent object advantage in natural scenes (Henderson, Weeks, \& Hollingworth, 1999; Hollingworth \& Henderson, 1998). For example, Hollingworth and Henderson asked participants to detect target objects in briefly presented line drawing scenes. The results showed that detection performance was better for objects that were semantically inconsistent with scene context (e.g., a mixer in a farmyard) than for objects that were consistent (e.g., a chicken in a farmyard). Recently, using the probe detection technique, Gordon (2004) demonstrated that semantically inconsistent objects affected the control of attentional deployment in the scene. Participants viewed briefly presented scenes followed by a probe dot appearing at the location of a consistent or an inconsistent object. The results showed that the response to a probe at the site of the inconsistent object was faster than that to a probe at the site of the consistent object. This result is important because it provides strong evidence that the semantic context of a scene can influence attentional allocation as well as display configurations learned in a contextual cuing task do. An important goal for future research is to examine the relationship between the mechanism of attentional allocations to the semantically inconsistent advantage and the mechanism of the new object advantage, which underlie the ecologically relevant control of visual attention in visual scenes encountered in everyday life.

\section{REFERENCES}

Awh, E., \& Pashler, H. (2000). Evidence for split attentional foci. Journal of Experimental Psychology: Human Perception \& Performance, 26, 834-846.

Brainard, D. H. (1997). The Psychophysics Toolbox. Spatial Vision, 10, 433-436. 
Castiello, U., \& Umiltà, C. (1992). Splitting focal attention. Journal of Experimental Psychology: Human Perception \& Performance, 18, 837-848.

Chun, M. M. (2000). Contextual cueing of visual attention. Trends in Cognitive Sciences, 4, 170-178.

Chun, M. M., \& JiAng, Y. (1998). Contextual cueing: Implicit learning and memory of visual context guides spatial attention. Cognitive Psychology, 36, 28-71.

FISER, J., \& AsLin, R. N. (2001). Unsupervised statistical learning of higher-order spatial structures from visual scenes. Psychological Science, 12, 499-504.

FISER, J., \& ASLIN, R. N. (2002). Statistical learning of higher-order temporal structure from visual shape sequences. Journal of Experimental Psychology: Learning, Memory, \& Cognition, 28, 458-467.

GodiJn, R., \& Theeuwes, J. (2003). Parallel allocation of attention prior to the execution of saccade sequences. Journal of Experimental Psychology: Human Perception \& Performance, 29, 882-896.

Gordon, R. D. (2004). Attentional allocation during the perception of scenes. Journal of Experimental Psychology: Human Perception \& Performance, 30, 760-777.

Hahn, S., \& Kramer, A. F. (1998). Further evidence for the division of attention between noncontiguous locations. Visual Cognition, 5, 217-256.

Henderson, J. M., Weeks, P. A., \& Hollingworth, A. (1999). The effects of semantic consistency on eye movements during complex scene viewing. Journal of Experimental Psychology: Human Perception \& Performance, 25, 210-228.

Hillstrom, A. P., \& Yantis, S. (1994). Visual motion and attentional capture. Perception \& Psychophysics, 55, 399-411.

Hollingworth, A., \& Henderson, J. M. (1998). Does consistent scene context facilitate object perception? Journal of Experimental Psychology: General, 127, 398-415.

Johnston, W. A., Schwarting, I. S., \& Hawley, K. J. (1996). Novel pop-out, perceptual inhibition, and the stability-plasticity dilemma. In A. F. Kramer, M. G. H. Coles, \& G. D. Logan (Eds.), Converging operations in the study of visual selective attention (pp. 315-335). Washington, DC: American Psychological Association.

JoNidEs, J., \& YANTIS, S. (1988). Uniqueness of abrupt visual onset in capturing attention. Perception \& Psychophysics, 43, 346-354.

KIM, M. S., \& CAve, K. R. (1995). Spatial attention in visual search for features and feature conjunctions. Psychological Science, 6, 376380 .

KLEIN, R. M. (1988). Inhibitory tagging system facilitates visual search. Nature, 334, 430-431.

Koshino, H., Warner, C. B., \& Juola, J. F. (1992). Relative effectiveness of central, peripheral, and abrupt-onset cues in visual attention. Quarterly Journal of Experimental Psychology, 45A, 609-631.

Kramer, A. F., \& HAHN, S. (1995). Splitting the beam: Distribution of attention over noncontiguous regions of the visual field. Psychological Science, 6, 381-386.
Maljkovic, V., \& Nakayama, K. (2000). Priming of pop-out: III. A short-term implicit memory system beneficial for rapid target selection. Visual Cognition, 7, 571-595.

Pelli, D. G. (1997). The VideoToolbox software for visual psychophysics: Transforming numbers into movies. Spatial Vision, 10, 437-442.

Peterson, M. S., \& Kramer, A. F. (2001a). Attentional guidance of the eyes by contextual information and abrupt onsets. Perception \& Psychophysics, 63, 1239-1249.

Peterson, M. S., \& Kramer, A. F. (2001b). Contextual cueing reduces interference from task-irrelevant onset distractors. Visual Cognition, 8, 843-859.

ReBer, A. S. (1989). Implicit learning and tacit knowledge. Journal of Experimental Psychology: General, 118, 219-235.

Reicher, G. M. (1969). Perceptual recognition as a function of meaningfulness of stimulus material. Journal of Experimental Psychology, 81, 274-280.

Saffran, J. R., Aslin, R. N., \& Newport, E. L. (1996). Statistical learning by 8-month-old infants. Science, 274, 1926-1928.

Theeuwes, J. (1994). Stimulus-driven capture and attentional set: Selective search for color and visual abrupt onsets. Journal of Experimental Psychology: Human Perception \& Performance, 20, 799-806.

Theeuwes, J., Kramer, A. F., Halm, S., \& Irwin, D. E. (1998). Our eyes do not always go where we want them to go: Capture of the eyes by new objects. Psychological Science, 9, 379-385.

VAn Selst, M., \& Jolicceur, P. (1994). A solution to the effect of sample size on outlier elimination. Quarterly Journal of Experimental Psychology, 47A, 631-650.

Watson, D. G., \& Humphreys, G. W. (1997). Visual marking: Prioritizing selection for new objects by top-down attentional inhibition of old objects. Psychological Review, 104, 90-122.

Watson, D. G., \& Humphreys, G. W. (2000). Visual marking: Evidence for inhibition using a probe-dot detection paradigm. Perception \& Psychophysics, 62, 471-481.

Weisstein, N., \& Harris, C. S. (1974). Visual detection of line segments: An object-superiority effect. Science, 186, 752-755.

YANTIS, S. (1993). Stimulus-driven attentional capture and attentional control settings. Journal of Experimental Psychology: Human Perception \& Performance, 19, 676-681.

Yantis, S., \& Hillstrom, A. P. (1994). Stimulus-driven attentional capture: Evidence from equiluminant visual objects. Journal of Experimental Psychology: Human Perception \& Performance, 20, 95-107.

YANTIS, S., \& Jonides, J. (1984). Abrupt visual onsets and selective attention: Evidence from visual search. Journal of Experimental Psychology: Human Perception \& Performance, 10, 601-621.

YANTIS, S., \& Jonides, J. (1990). Abrupt visual onsets and selective attention: Voluntary versus automatic allocation. Journal of Experimental Psychology: Human Perception \& Performance, 16, 121-134.

(Manuscript received January 11, 2005; revision accepted for publication October 28,2005 .) 